\title{
Propiedades Psicométricas de la Versión Española del Teamwork Skills Questionnaire (TSQ)
}

\author{
Psychometric Properties of the Spanish Version of the Teamwork Skills \\ Questionnaire (TSQ)
}

\author{
Iago Portela Pino ${ }^{1}$, José Domínguez Alonso ${ }^{2}$ y Margarita Pino Juste ${ }^{3}$
}

\begin{abstract}
Resumen
El objetivo principal del estudio es traducir y adaptar el cuestionario de habilidades de trabajo en equipo (TSQ) al español, analizar las propiedades psicométricas de esta adaptación, y alcanzar evidencias de validez (corroborar la estructura factorial original y su validez convergente), y de fiabilidad (consistencia interna). Participaron 615 alumnos con una media de edad de 21.52 años $(\mathrm{DT}=3.73)$. Los resultados ratifican la estructura factorial del cuestionario original compuesto por 36 ítems y seis factores (adaptabilidad, coordinación, toma de decisiones, liderazgo, destrezas interpersonales y comunicación $)(\chi 2 / \mathrm{gl}=3.67$, $\mathrm{CFI}=.937, \mathrm{NNFI}=.890, \mathrm{RMSEA}=.056)$ y una consistencia interna muy elevada $(\alpha=.938)$. Los seis factores se asociaron directa y moderadamente entre ellos ( $\mathrm{r}$ entre .423 y .733), excepto entre destrezas interpersonales y liderazgo ( $\mathrm{r}=.179)$ que fue baja. En conclusión, este estudio aporta nuevos datos sobre una herramienta válida y fiable para evaluar las habilidades de trabajo en equipo.
\end{abstract}

Palabras clave: competencia transversal, habilidades blandas, trabajo en equipo, educación superior

\begin{abstract}
The main objective of the study is to translate and adapt the Teamwork Skills Questionnaire (TSQ) into Spanish, analyze the psychometric properties of this adaptation, and reach evidence of validity (corroborating the original factor structure and its convergent validity), and reliability (internal consistency). The results confirm the factor structure of the original questionnaire composed of 36 items and six factors (adaptability, coordination, decision making, leadership, interpersonal skills and communication) $(\chi 2 / \mathrm{gl}=3.67, \mathrm{CFI}=.937, \mathrm{NNFI}=.890, \mathrm{RMSEA}=.056)$ and a very high internal consistency $(\alpha=.938)$. The six factors were directly and moderately associated with each other ( $\mathrm{r}$ between .423 and .733), except between interpersonal skills and leadership $(\mathrm{r}=.179)$ that which was low. In conclusion, this study provides new data on a valid and reliable tool to assess teamwork skills.
\end{abstract}

Keywords: transversal competence, soft skills, teamwork, higher education

\footnotetext{
${ }^{1}$ Dr. en Ciencias del Deporte. Profesor Ayudante Doctor. Departamento de Ciencias del Deporte. Universidad Internacional Isabel I. C/ Fernán González, 76, 09003. 689038142 Burgos, España. Correo: iagoport92@gmail.com

${ }_{2}$ Psicólogo. Dr. en Psicología. Profesor Asociado. Universidad de Vigo. Departamento de Ciencias del Comportamiento. Campus As Lagoas, 32004 Ourense, España. Tel.: 988387100. Correo: jdalonso@uvigo.es

3 Dra. en Ciencias de la Educación. Catedrática de Universidad. Departamento de Didáctica, Organización Escolar y Métodos de Investigación. Universidad de Vigo. Campus A Xunqueira, 36005 Pontevedra, España. Tel.: 986801723. Correo: mpino@uvigo.es

Revista Iberoamericana de Diagnóstico y Evaluación - e Avaliação Psicológica. RIDEP · No62 · Vol.1 · 155-165 · 2022

ISSN: 1135-3848 print /2183-6051online
} 


\section{Introducción}

Las habilidades de trabajo en equipo, comúnmente denominadas habilidades blandas, son aquellas relacionadas con la capacidad de una persona para trabajar y colaborar eficazmente con otros cuando se le asigna como miembro de un equipo específico. Normalmente estas habilidades están ligadas al concepto de competencia y a otros como cualificación, especialización, productividad, rendimiento, utilidad, practicidad, habilidad, pericia y destreza, entre otros. El concepto de competencia tiene como punto de partida una comprensión economicista de la sociedad, relacionado de forma expresa con los conceptos de preparación y perfeccionamiento de cualquier profesional (Silva Carreño \& Mazuera Moreno, 2019).

Estas son habilidades como la coordinación, la comunicación, la resolución de conflictos, toma de decisiones, negociación o la adaptabilidad. Kautz et al. (2014) señalan que estas habilidades se pueden medir, ya que son estables a cualquier edad en diferentes tareas, pero pueden cambiar a lo largo del ciclo de vida. La habilidad es un proceso formativo que se lleva a cabo en el ámbito familiar, escolar y social. Su desarrollo y aprendizaje es considerado como un proceso dinámico, en el que los primeros años sientan las bases para una inversión exitosa en periodos posteriores.

En los últimos diez años han aparecido, en diferentes bases de datos, estudios que muestran a estas habilidades blandas como esenciales para el desempeño laboral en una economía globalizada. De hecho, son las más buscadas en el mercado laboral y muy difíciles de detectar en un currículum. Las empresas en Estados Unidos dicen que cada vez es más difícil encontrar solicitantes que puede comunicarse claramente con sus compañeros, tener iniciativa, resolver problemas o simplemente llevarse bien con sus compañeros de trabajo (Davidson, 2016). En consecuencia, las empresas requieren cada vez más de sus trabajadores que asuman responsabilidades que exigen pensamiento crítico, empatía u otras habilidades sociales que los ordenadores no pueden simular.

Es decir, para poder desempeñarse con éxito como ciudadano del siglo XXI, es necesario contar con habilidades cognitivas, así como intra e interpersonales. El éxito también significa poder cumplir nuestras metas personales y contar con las competencias necesarias para influir en el mundo que nos rodea, por lo cual es de suma importancia desarrollar una conciencia clara de nosotros mismos, de nuestras capacidades y de la amplia gama de opciones que tenemos para elegir, así como de las competencias que requerimos para poder transitar con éxito (perseverancia) por los caminos elegidos y ser capaces de tomar buenas decisiones en el futuro, como ciudadanos comprometidos con el mundo (Friedlaender et al., 2014; Nagaoka et al., 2015). En gran medida nuestras habilidades sociemocionales de perseverancia y toma de decisiones están vinculadas, fundamentalmente con la conciencia y regulación de nuestras emociones.

Revisiones sistemáticas sobre el tema, identificaron habilidades blandas que fomentan resultados positivos en la fuerza laboral, pero también para la prevención de la violencia y la salud sexual y reproductiva (Gates et al., 2016). En concreto, Robles (2012) identificó diez habilidades blandas como las de mayor relevancia para los empleadores: integridad, comunicación, cortesía, responsabilidad, habilidades sociales, actitud positiva, profesionalismo, flexibilidad, trabajo en equipo y ética laboral. En esta línea, estudios como los realizados por GonzálezMorales et al. (2010) o Penzenstadler et al. (2013), coinciden en señalar a la capacidad de análisis, habilidad comunicativa o sensibilidad frente a problemas del cliente, como las más solicitadas en el ámbito laboral (Tabla 1).

El estudio de Gutman y Schoon (2013) ha identificado las siguientes habilidades blandas: competencias intrapersonales (flexibilidad, iniciativa, apreciación de la diversidad y metacognición), y competencias interpersonales (comunicación, colaboración, responsabilidad y resolución de conflictos). En consonancia, el National Research Council (NRC) y la National Academy of Sciences (NAS) de los Estados Unidos organizaron las habilidades del siglo XXI en tres categorías descritas por Kyllonen (2015) de la siguiente forma: habilidades cognitivas (pensamiento crítico, solución de problemas y creatividad); habilidades interpersonales (habilidades de comunicación, habilidades sociales, 
Tabla 1. Conjunto de habilidades blandas más solicitadas en el ámbito laboral

\begin{tabular}{ll}
\hline Propuesta de González-Morales et al. (2010) & Propuesta de Penzenstadler et al. (2013) \\
\hline Búsqueda y clasificación de información & Habilidades de abstracción \\
Redacción de informes/Trabajo en equipo & Habilidades de creatividad \\
Liderazgo y supervisión/ Toma de decisiones & Capacidad de análisis $(*)$ \\
Gestión de conflictos/Pensamiento crítico & Habilidades comunicativas $(*)$ \\
Gestión de clientes y de las expectativas del cliente & Sensibilidad frente a los problemas del cliente $(*)$ \\
Evaluación y comunicación de resultados & \\
\hline Nota. Las habilidades de la propuesta de Penzenstadler et al. (2013) & marcadas con $(*)$ están incluidas también en la propuesta de González- \\
Morales et al. $(2010)$.
\end{tabular}
Morales et al. (2010).

trabajo en equipo, sensibilidad cultural y lidiar con la adversidad); y, habilidades intrapersonales (autogestión, autorregulación, gestión del tiempo, autodesarrollo -aprendizaje permanente-, adaptabilidad y funcionamiento ejecutivo).

No obstante, el estudio de las habilidades blandas se ve limitado, además de por la falta de claridad en la definición de los términos, por la mala medición (Chetty et al., 2011; Deming, 2009; 2017; Matteson et al., 2016). Normalmente se utilizan dos tipos de enfoques para medir estas habilidades: directos e indirectos. Entre los primeros está la observación que parece ser un proceso demasiado largo y complejo o las simulaciones por ordenador, pero no resultan rentables en muchos entornos o no son factibles. Por tanto, O'Neil et al. (1999) desarrollaron un método indirecto mediante un cuestionario de autoinforme de 10 minutos para medir las habilidades de trabajo en equipo de los individuos sin tener que identificar a todas las personas que están participando en el equipo (Hsieh \& O'Neil, 2002; Marshall et al., 2005; O’Neil et al., 2000). El cuestionario parece ser el método más satisfactorio en términos de equilibrio entre practicidad, confiabilidad y validez, y facilidad de administración (Brungardt, 2009). Autores como Kautz et al. (2014) señalan sobre la medición y el aumento de las habilidades cognitivas y no cognitivas que las pruebas de rendimiento no miden adecuadamente los rasgos de personalidad, objetivos, motivaciones y preferencias que se valoran en el mercado laboral, en la escuela y en muchos otros ámbitos. Existen instrumentos que miden la efectividad del trabajo en equipo, pero no se centran en las diferentes habilidades que conforman este constructo (Delgado-Abella et al., 2020).

En consecuencia, pese a las limitaciones, este cuestionario denominado Teamwork Skills Questionnaire (TSQ) ha sido adopt ado por instituciones de reconocido prestigio como el
UCLA's National Center for Research on Evaluations, Standards, and Student Testing, commonly referred to as CRSST (O'Neil et al., 1997). Las seis habilidades de trabajo en equipo que se evalúan son coordinación, toma de decisiones, Liderazgo, habilidades interpersonales, adaptabilidad y comunicación (O'Neil et al., 1997, p. 413). El análisis en varias poblaciones de la consistencia interna de la escala mediante el alpha de Cronbach oscila habitualmente entre .84 y .97 (Chen, 2002; Hsieh, 2001; Kuehl, 2001; Marshall et al., 2005; O’Neil et al., 2003). Aun cuando este modelo ha sido utilizado en los EEUU, su aplicación en Europa y Latinoamérica sigue siendo un área emergente de investigación y está fragmentado en diferentes disciplinas. De ahí la importancia de disponer de una herramienta en español que permita a los empleadores medir el dominio de estas habilidades en sus empleados en los procesos de selección, pero también para el diseño de actividades formativas durante la etapa laboral. También puede ser útil para los profesores de secundaria, formación profesional y de universidad ya que como se ha dejado patente las habilidades blandas por su importancia para la inserción laboral están dentro del curriculum como competencias transversales, pero muy pocas veces se evalúan.

Por lo tanto, el objetivo de esta investigación es traducir y adaptar el TSQ al español, analizar sus propiedades psicométricas, y obtener evidencias de validez (análisis de la estructura factorial original y validez convergente), y de fiabilidad (consistencia interna) de manera que pueda ser utilizado tanto en el ámbito educativo como laboral. 


\section{Método}

\section{Participantes}

Se ha seleccionado la muestra mediante un muestreo aleatorio simple eligiendo el $25 \%$ de los estudiantes de cada curso y facultad en función del número asignado en su expediente y se le enviaba el formulario por correo electrónico. Participaron en total 615 alumnos de educación superior en el ámbito de las Ciencias sociales de la Comunidad Autónoma de Galicia. Su edad cronológica osciló entre 18 y 43 años $(\mathrm{M}=21.52$; DT=3.73). El 67\% de la muestra son mujeres y el $33 \%$ hombres. Según el grado universitario, un $31.4 \%$ cursa primero, $26 \%$ segundo, $25.5 \%$ tercero, y el $17.1 \%$ cuarto. El $54.5 \%$ son de la titulación de educación (magisterio y pedagogía), y el $45.5 \%$ de la titulación de gestión (administración y dirección de empresas y turismo).

\section{Instrumento}

La escala utilizada es el Teamwork Skills Questionnaire (TSQ) para la medición de hábitos de habilidades de trabajo en equipo teniendo en cuenta que el método más directo para la medición de la fuerza del hábito es pedir a los sujetos informar directamente sobre sus hábitos. Se entiende el hábito como un constructo psicológico que va más allá que la simple frecuencia del comportamiento pasado (Verplanken, 2006). Fundamentalmente los avances en la teoría del hábito vienen marcados por la distinción de hábito de la frecuencia de su ocurrencia y el uso de medidas independientes de estas dos construcciones (Verplanken \& Orbell, 2003; Orbell \& Verplanken, 2015).

Todas las medidas fueron codificadas de manera que los valores altos indican fuertes hábitos en lo referente a habilidades que una persona tiene para ser eficaz durante un trabajo en equipo y los valores más bajos lo contrario. Se ha operado con la escala de cinco grados de intensidad en el continuo aceptación-rechazo o acuerdo-desacuerdo, asignamos el valor 4 «casi siempre», 3 «a menudo», 2 «A veces» y 1 «Casi nunca», tal como se realiza en la escala original (O’Neill et al., 1999).

El cuestionario tiene seis factores: (a) coordinación, (b) toma de decisiones, (c) liderazgo, (d) habilidades interpersonales, (e) adaptabilidad y (f) comunicación (O'Neil et al., 1997, p. 413). Por coordinación (ítems: 6, 11, 17, 23,32 ) se entiende el dominio de un sujeto para organizar las actividades del equipo con el fin de completar una tarea a tiempo. Por toma de decisiones (ítems: $3,7,12,18,24,28$ ) se entiende el dominio en la utilización de la información disponible para tomar decisiones de equipo. Por liderazgo (ítems: 1, 4, 8, 13, 19, 25, 29) se entiende las capacidades de dirección de un equipo. Las habilidades interpersonales (ítems: 5, $9,14,20,33,36)$ se relacionan con la capacidad de interactuar cooperativamente con otros miembros del equipo. La adaptabilidad (ítems: 15 , $21,26,30,34)$ se refiere a poder reconocer problemas y responder adecuadamente durante la actividad laboral. La comunicación (ítems: 2, 10, $16,22,27,31,35)$ es el intercambio global de información clara y precisa.

\section{Procedimiento}

La traducción de la TSQ se realizó de manera inversa (Brislin, 1970; 1986) de tal forma que en primer lugar los ítems se tradujeron al castellano por un hablante nativo bilingüe de inglés, para posteriormente ser traducidos al inglés por un traductor ajeno al grupo de investigación (Hambleton, 1996; Muñiz et al., 2013). Ambos traductores fueron informados previamente sobre la escala y el tipo de respuestas utilizadas.

Se compararon los ítems traducidos al inglés con los ítems de la escala original observándose una total coincidencia, salvo en dos casos de conceptos muy concretos. En estos dos casos se decidió introducir los dos términos en la escala final, uno de ellos entre paréntesis a modo de aclaración, para facilitar la comprensión en caso de duda.

Una vez traducida la escala fue pasada a una muestra pequeña (25 alumnos) para verificar la correcta comprensión de los ítems. Este proceso dio lugar a pequeños cambios sintácticos en la redacción de alguna frase. Se ha tenido también en cuenta el sesgo de referencia dado que los estudiantes podrían responder teniendo en cuenta las ideas generadas en su contexto (Heckman \& Kautz, 2013), pero se controla con una información precisa de lo que se entiende por cada habilidad. 
Todos los sujetos participaron de forma voluntaria y anónima en el estudio y se respetaron todos los procedimientos éticos para la recogida de datos. La administración del instrumento a los estudiantes universitarios se realizó mediante un formulario en Google Drive enviado por correo electrónico, haciendo constar la participación anónima, voluntaria y la confidencialidad de la información. Así como el objetivo de la investigación y se hizo hincapié en la importancia de la sinceridad de las respuestas.

\section{Análisis de datos}

Se ha calculado para cada ítem de la escala, su media, desviación típica, correlación ítem-total, alfa de Cronbach si se elimina un elemento, asimetría y curtosis. Se ha considerado para asimetría y curtosis los valores de rango entre 1.50 y +1.50 como adecuados (Forero et al., 2009). Siguiendo las recomendaciones de Ledesma et al. (2019) se testó la estructura del modelo original de seis factores, a través del uso secuencial del AFE y AFC.

Así, con el primer subgrupo de participantes $(n=315)$ se lleva a cabo el AFE (método de Máxima Verosimilitud con rotación varimax debido a ser una técnica multivariante que permite el tratamiento conjunto de las variables observadas reduciendo así el número de datos y consiguiendo identificar un grupo de variables a partir de las inicialmente observadas-) para identificar el número de factores latentes. A continuación, con el segundo grupo de participantes $(n=300)$ se realizó el AFC (Mínimos Cuadrados Generalizados) para testear la validez del modelo. En la evaluación de modelos, se considera un ajuste bueno o satisfactorio si los índices de ajuste global: $\mathrm{X}^{2} / \mathrm{gl}$ (estadístico de chicuadrado dividido por los grados de libertad) oscila entre 2.0 y 5.0; CFI (Comparative fit index)

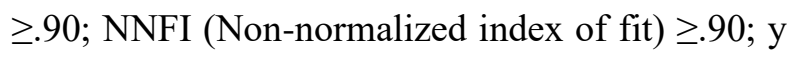
RMSEA (Root mean squared error of approximation) <.08 (Byrne, 1989; 2010; Hu \& Bentler, 1999; Steiger, 2007).

Finalmente, la confiabilidad se obtiene a través del Alfa de Cronbach (programa SPSS) y Omega de McDonald (programa Excel). Para el análisis de los datos se emplearon los programas estadísticos SPSS 22.0 y AMOS v.24.

\section{Resultados}

\section{Análisis univariantes}

En la Tabla 2 se muestran los estadísticos descriptivos, asimetría y curtosis obtenidos tras la aplicación del cuestionario. La correlación ítemtotal corregida ( $\mathrm{r}_{\mathrm{i}-\mathrm{t}}$ entre .409 y .648) es positiva en todos los ítems, lo que indica que todos contribuyen en la misma dirección a medir el constructo teórico de referencia.

También se puede observar que las puntuaciones obtenidas en cada enunciado (ítem) del cuestionario Teamwork Skills Questionnaire en los índices de asimetría univariante (oscilaron entre -1.42 y .40) y curtosis univariante (oscilaron entre -.82 y 1.42). Se evidencia, por tanto, que todos los ítems presentan un ajuste aceptable a la distribución normal. Asimismo, el Alfa de Cronbach si se elimina un elemento (todos los valores inferiores a la fiabilidad del conjunto de la prueba $\alpha_{\mathrm{T}}=.938$ ) muestra que la supresión de cualquiera de los ítems no mejoraría la fiabilidad del conjunto del cuestionario.

\section{Estructura factorial exploratoria y confirmatoria}

Previamente (submuestra: $n=315$ ), se llevaron a cabo las medidas de adecuación muestral requeridas, obteniendo un índice de .951 en la prueba de Kaiser-Meyer-Olkin, el cual se considera excelente para llevar a cabo el análisis factorial (Beavers et al., 2013). Además, la prueba de esfericidad de Bartlett $(p<.001)$ confirma la posibilidad de extraer factores de la matriz de correlaciones. Así pues, se utiliza un análisis factorial de componentes principales con rotación varimax (forzado a seis factores del cuestionario original), adoptando como criterios de inclusión en cada factor: insertar cada ítem en un solo factor, establecer estimaciones de .30 como valor mínimo de saturación (Ferrando \& Anguiano, 2010) e incluir el ítem que satura en más de un factor en el factor determinado por el cuestionario original. Reseñar que todos sus enunciados presentan comunalidades entre .415 (ítem 7) y .704 (ítem 1), es decir, superiores a .40. Los seis factores del AFE explican el 55.68\% de la varianza total de los datos y replican la estructura propuesta por los autores, a excepción de los ítems 23, 22, 24, 31, 34 que saturan en más de un factor. 
Tabla 2. Estadísticos descriptivos y capacidad discriminativa de los ítems de la escala TSQ

\begin{tabular}{|c|c|c|c|c|c|c|c|}
\hline SUBESCALA & ITEM & MEDIA & DT & $\mathrm{r}_{\mathrm{i}-\mathrm{t} *}$ & $\begin{array}{l}\text { ASIMETRÍA } \\
\text { (E.T.=.099) }\end{array}$ & $\begin{array}{l}\text { CURTOSIS } \\
\text { (E.T.=.197) }\end{array}$ & $\begin{array}{c}\alpha \text { si se elimina } \\
\text { elemento }\end{array}$ \\
\hline \multirow{5}{*}{ Adaptabilidad } & 15 & 2.95 & .746 & .509 & -.131 & -.679 & .936 \\
\hline & 21 & 3.28 & .684 & .626 & -.541 & -.284 & .935 \\
\hline & 26 & 3.14 & .749 & .588 & -.429 & -.536 & .935 \\
\hline & 30 & 3.06 & .739 & .499 & -.236 & -.718 & .936 \\
\hline & 34 & 2.97 & .728 & .648 & -.184 & -.514 & .935 \\
\hline \multirow{5}{*}{ Coordinación } & 6 & 2.81 & .891 & .453 & -.304 & -.678 & .937 \\
\hline & 11 & 3.16 & .808 & .526 & -.624 & -.355 & .936 \\
\hline & 17 & 3.26 & .783 & .465 & -.697 & -.409 & .937 \\
\hline & 23 & 2.51 & .906 & .496 & .104 & -.786 & .936 \\
\hline & 32 & 3.21 & .822 & .507 & -.691 & -.416 & .936 \\
\hline \multirow{6}{*}{ Toma de decisiones } & 3 & 3.17 & .725 & .573 & -.529 & -.114 & .936 \\
\hline & 7 & 2.92 & .830 & .540 & -.319 & -.587 & .936 \\
\hline & 12 & 2.95 & .759 & .591 & -.140 & -.721 & .935 \\
\hline & 18 & 2.88 & .789 & .583 & -.260 & -.435 & .935 \\
\hline & 24 & 3.25 & .740 & .564 & -.657 & -.176 & .936 \\
\hline & 28 & 3.16 & .745 & .582 & -.426 & -.577 & .936 \\
\hline \multirow{7}{*}{ Liderazgo } & 1 & 2.65 & .862 & .440 & .111 & -.806 & .937 \\
\hline & 4 & 2.51 & .803 & .451 & .400 & -.495 & .937 \\
\hline & 8 & 2.46 & .832 & .499 & .219 & -.521 & .936 \\
\hline & 13 & 2.85 & .937 & .594 & -.344 & -.827 & .935 \\
\hline & 19 & 2.67 & .828 & .546 & -.041 & -.616 & .936 \\
\hline & 25 & 2.73 & .894 & .550 & -.174 & -.764 & .936 \\
\hline & 29 & 3.07 & .818 & .594 & -.455 & -.587 & .935 \\
\hline \multirow{6}{*}{$\begin{array}{l}\text { Destrezas } \\
\text { interpersonales }\end{array}$} & 5 & 3.47 & .710 & .540 & -1.194 & .816 & .936 \\
\hline & 9 & 3.47 & .714 & .426 & -1.213 & .906 & .937 \\
\hline & 14 & 3.53 & .652 & .410 & -1.191 & .688 & .937 \\
\hline & 20 & 3.53 & .691 & .460 & -1.362 & 1.239 & .937 \\
\hline & 33 & 3.29 & .732 & .445 & -.764 & .097 & .937 \\
\hline & 36 & 3.49 & .728 & .409 & -1.421 & 1.421 & .937 \\
\hline \multirow{7}{*}{ Comunicación } & 2 & 3.05 & .827 & .516 & -.528 & -.367 & .936 \\
\hline & 10 & 3.30 & .723 & .536 & -.781 & .204 & .936 \\
\hline & 16 & 3.36 & .683 & .572 & -.727 & -.107 & .936 \\
\hline & 22 & 3.26 & .718 & .646 & -.670 & .037 & .935 \\
\hline & 27 & 3.51 & .645 & .481 & -1.116 & .751 & .936 \\
\hline & 31 & 3.18 & .706 & .622 & -.488 & -.114 & .935 \\
\hline & 35 & 3.48 & .675 & .527 & -1.123 & .792 & .936 \\
\hline
\end{tabular}

* $\mathrm{r}_{\mathrm{i}-\mathrm{t}}$ correlación item-total corregida

A continuación (submuestra: $n=300$ ), para ratificar la orientación de los ítems y verificar las relaciones entre los seis factores, nos hemos basado en la distribución obtenida por O'Neil et al. (1997) que es similar a la alcanzada con el AFE de la muestra actual. Se propuso el modelo inicial de medida establecido por O'Neil et al. (1997) (Figura 1), que consta de 6 variables latentes (A: adaptabilidad, CO: coordinación, TD: toma de decisiones, L: liderazgo; DI: destrezas interpersonales, C: comunicación) y 36 variables observadas (ítems). Al especificar el modelo se observan cargas factoriales satisfactorias en todos los ítems (valores superiores a .50 indicado por Byrne, 2010), por lo cual no fue eliminado ningún ítem.
Al especificar el modelo, y comprobada la normalidad multivariada (coeficiente de Mardía = 226.67) inferior al valor alcanzado al utilizar la expresión $\mathrm{px}(\mathrm{p}+2)$, siendo $\mathrm{p}$ el número de variables observadas [36x $(36+2)=1.368]$, de acuerdo con Bollen (1989). Así pues, se procedió a la estimación de parámetros por el procedimiento de máxima verosimilitud (ML) por ser el más eficiente y no sesgado cuando se cumplen los supuestos de normalidad multivariante.

En consecuencia, se ponen a prueba dos modelos de medida alternativos (Tabla 3). Un primer modelo de seis factores hipotetizados a partir de los 36 ítems del instrumento original (M6F). Un segundo modelo unifactorial también 


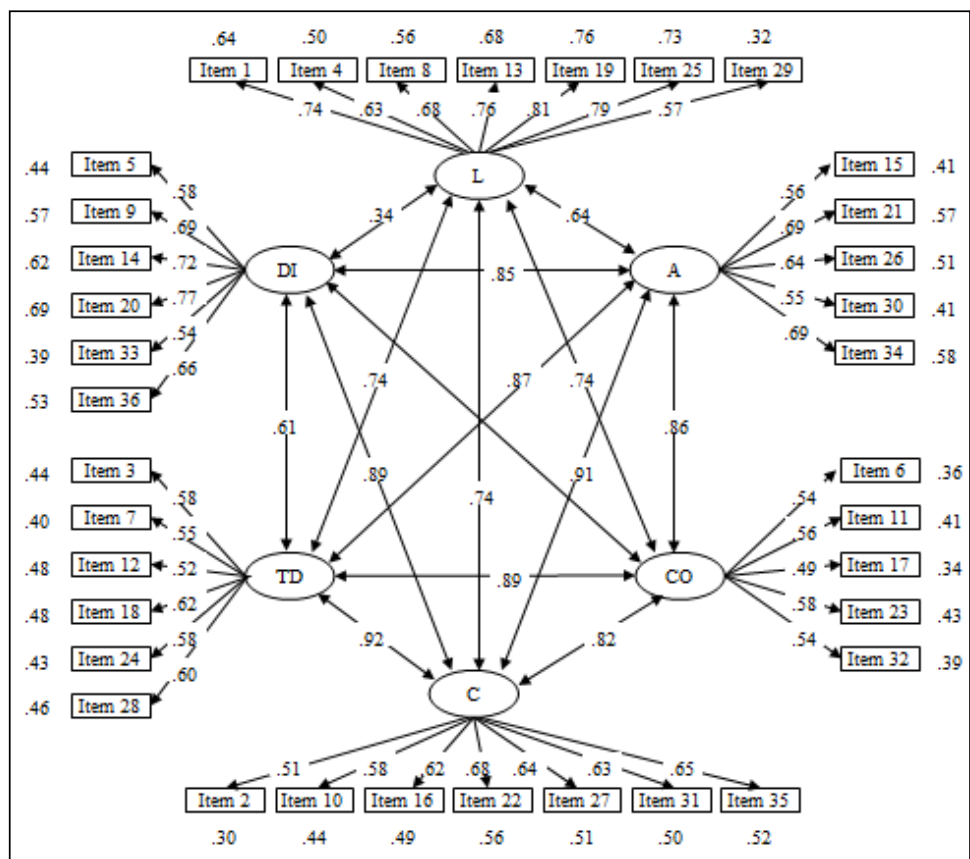

Figura 1. Estructura factorial del M6F (A: adaptabilidad, CO: coordinación, TD: toma de decisiones, L: liderazgo; DI: destrezas interpersonales, C: comunicación)

Tabla 3. Índices de bondad de ajuste de los modelos de seis factores (M6F) y unifactorial (M1F) del cuestionario TSQ con la segunda muestra $(n=300)$

\begin{tabular}{llllllllll}
\hline Modelo & S-Bx2 & g.l. & X2/gl & RMSEA (IC 90\%) & NNFI & CFI & $\Delta$ RMSEA & $\Delta$ NNFI & $\Delta$ CFI \\
\hline M1F & $3793.15^{* *}$ & 595 & 6.38 & $.084(.081-.087)$ & .727 & .764 & \multirow{2}{*}{028} & .163 & .173 \\
M6F & $2129.81^{* *}$ & 579 & 3.67 & $.056(.053-.059)$ & .890 & .937 & & .163 & \\
\hline
\end{tabular}

** $p=.000$; g.l.=grados de libertad; IC=intervalo de confianza; M1F=Modelo unifactorial; M6F=Modelo de seis factores.

Tabla 4. Análisis correlacional entre las dimensiones y Alpha de Cronbach del TSQ

\begin{tabular}{|c|c|c|c|c|c|c|}
\hline TSQ & 1 & 2 & 3 & 4 & 5 & Alpha \\
\hline 1. Adaptabilidad & - & & & & & .767 \\
\hline 2. Coordinación & $.607 * *$ & - & & & & .665 \\
\hline 3. Toma decisiones & $.729 * *$ & $.708 * *$ & - & & & .762 \\
\hline 4. Liderazgo & $.567 * *$ & $.594 * *$ & $.645^{* *}$ & - & & .868 \\
\hline 5. Destrezas interpersonales & $.534 * *$ & $.423 * *$ & $.519 * *$ & $.179 * *$ & - & .814 \\
\hline 6. Comunicación & $.696 * *$ & $.622 * *$ & $.733 * *$ & $.481 * *$ & $.724 * *$ & .800 \\
\hline
\end{tabular}

con los 36 ítems de la escala inicial (M1F). Los resultados obtenidos indican que el M6F es el que mejor bondad de ajuste ofrece $(\chi 2 / \mathrm{gl}=3.67$, $\mathrm{CFI}=.937, \mathrm{NNFI}=.890, \mathrm{RMSEA}=.056)$. Así, los índices de ajuste en el M6F son adecuados, con valores más bajos en $\chi 2 / g 1$ y RMSEA, y superiores en GFI y AGFI que en el modelo unifactorial (M1F).

\section{Validez concurrente y fiabilidad}

Las correlaciones entre las seis dimensiones que conforman la escala TSQ fueron positivas y medio-altas ( $\mathrm{r}$ entre .423 y .729), excepto la asociación entre el liderazgo y las destrezas interpersonales que fue muy baja $(r=.179)$. No obstante, todas las asociaciones fueron estadisticamente significativas a nivel de .001 (Tabla 4).

Asimismo, la fiabilidad del cuestionario, analizada en términos de consistencia interna, se calculó a través del coeficiente Alfa de Cronbach de cada factor. Se puede observar que los índices de fiabilidad de los factores son moderados, entre .762 (Toma de decisiones) y .868 (Liderazgo). Sólo en el factor coordinación (.665) muestra un alfa de Cronbach inferior a .70. Además, el cuestionario en su totalidad presenta una alta fiabilidad $(\alpha=.938 ; \omega=.944)$. 


\section{Discusión y conclusiones}

La competencia de trabajo en equipo es muy valorada en el contexto laboral y se revela como un elemento importante ya que conlleva las capacidades de organización y gestión primordiales para los empleadores (Dieste et al., 2019). Por tanto, disponer de un instrumento válido y fiable parece un objetivo prioritario. El objetivo de este trabajo ha sido contrastar la validez y fiabilidad del Teamwork Skills Questionnaire, un autoinforme diseñado para medir hábitos de habilidades de trabajo en equipo, basándose en el modelo propuesto por O'Neil et al. (1997). Los resultados alcanzados evidencian que el cuestionario TSQ es un instrumento con adecuadas propiedades psicométricas, y por tanto adecuado para ser utilizado para tal fin.

El presente estudio se propuso dar continuidad a la fiabilidad y validez de la estructura factorial de la versión original del cuestionario. En un primer momento, a través del análisis de los ítems que constituye la totalidad del cuestionario, se confirma la incorporación de todos los ítems del mismo. A continuación, se observa que la puntuación obtenida en cada ítem correlaciona positivamente con la alcanzada en el total de la escala, lo cual señala que no es necesario redireccionar en ninguno de sus enunciados el valor de las opciones de respuesta asignadas. Finalmente, la mayoría de los ítems muestran asimetría negativa (excepto los ítems: 1. Cuando trabajo en equipo, ejercito mi capacidad de liderazgo; 4. Cuando trabajo en equipo yo enseño a otros miembros del equipo; y 8. Cuando trabajo en equipo sirvo como modelo en las interacciones informales $y$ formales), lo cual revela una predisposición a alcanzar valores altos en la prueba. Es decir, los individuos informan que en su vida cotidiana desarrollan las habilidades de trabajo en equipo evaluadas en el cuestionario, excepto el ejercer capacidad de liderazgo, enseñar a otros miembros o servir como modelo cuando se trabaja en equipo.

Los análisis factoriales realizados muestran, de acuerdo a lo esperado (O'Neil et al., 1997) que el constructo "hábitos de trabajo en equipo" es multidimensional, es decir, está integrado por diferentes componentes o dimensiones. De hecho, los análisis exploratorios y confirmatorios realizados sostienen su estructura original de seis factores en la valoración de los hábitos de trabajo en equipo: adaptabilidad, coordinación, toma de decisiones, liderazgo, destrezas interpersonales y comunicación. Dichos resultados están en la línea de lo logrado inicialmente en el diseño original del cuestionario. Los seis factores hallados permiten explicar un porcentaje alto de la varianza.

Mediante el análisis factorial confirmatorio, se comprobó el ajuste hipotético al modelo original de seis factores y se comparó con un modelo unifactorial. Los datos confirman que el modelo de seis factores (M6F) presenta un ajuste satisfactorio a los datos empíricos $[\chi 2 / \mathrm{g}]=3.67$; $\mathrm{CFI}=.937$; NNFI=.890; RMSEA=.056], mejor que el modelo unifactorial. De hecho, el modelo unifactorial no confirma su estructura, ya que los valores de los índices absolutos (X2/g1 y RMSEA) como los índices incrementales (NNFI y CFI), presentaron valores inaceptables.

La consistencia interna fue medida mediante el alfa de Cronbach, ofreciendo una excelente estimación de fiabilidad del instrumento (Alfa de Cronbach=.938). Asimismo, los coeficientes obtenidos para cada una de las seis dimensiones de la prueba son óptimos [El alfa de Cronbach oscila entre $\quad .665$ (coordinación) y $\quad .868$ (liderazgo)]. Estos valores están en la misma línea de los trabajos realizados con poblaciones diversas por Brungardt (2009), Chen (2002), Hsieh (2001), Marshall et al. (2005), o O'Neil et al. (2003).

Además, las correlaciones entre los factores que conforman el constructo "hábitos de trabajo en equipo" variaron de moderada a fuerte magnitud (correlaciones en el rango de .423 a .733), con excepción de la obtenida entre los factores destrezas interpersonales y liderazgo, que es muy débil $(\mathrm{r}=.179)$. Ello lleva a sostener que hay grados razonables de separación entre las puntuaciones alcanzadas en los seis factores, con resultados similares a los alcanzados por Brungardt (2009).

En definitiva, el adecuado ajuste a la estructura factorial obtenida en el cuestionario original, confirma la validez y fiabilidad del modelo de seis factores para ser utilizado en poblaciones españolas.

La disponibilidad de una herramienta para medir habilidades blandas es importante, tanto en 
el ámbito laboral como educativo, ya que permite conocer el dominio de las mismas de un individuo concreto o de un grupo de personas. Por tanto, se podrán diseñar programas formativos que mejoren estas habilidades tanto en la formación inicial como permanente.

Hemos de tener en cuenta que, aunque la población es amplia para este tipo de estudios, pueden existir sesgos derivados de los datos auto informados recogidos mediante una aplicación informática y la no aleatorización previa de la muestra. Por tanto, no podemos excluir que puedan existir factores desconocidos que expliquen los resultados descriptivos (Price \& Murnan, 2004).

\section{Referencias}

Beavers, A. S., Lounsbury, J. W., Richards, J. K., Huck, S.W., Skolits, G. J., \& Esquivel, S. L. (2013). Practical considerations for using exploratory factor analysis in educational research. Practical Assessment, Research \& Evaluation, 18(6). Recuperado de http://pareonline.net/getvn.asp?v=18\&n=6.

Brislin, R. W. (1986). The wording and translation of research instruments. En W. L. Lonner and J. W. Berry (Eds.), Field Methods in Cross-Cultural Research Cross-Cultural Research and Methodology Series, 8 (pp. 137164). Sage.

Brislin, R.W. (1970). Back-translation for crosscultural research. Journal of Cross Cultural Psychology, 1, 187-196. https://doi.org/10.1177/135910457000100301

Brungardt, C. J. (2009). College graduates' perceptions of their use of teamwork skills: Soft skill development in fort hays state university leadership education. Doctoral dissertation. Kansas State University.

Byrne, B. M. (1989). A primer of lisrel: Basic applications and programming for confirmatory factor analytic models. New York: Springer-Verlag. https://doi.org/10.1007/978-1-4613-8885-2

Byrne, B. M. (2010). Structural equation modeling with AMOS (2nd ed.). Routledge.

Chen, H. (2002). Relationships of teamwork skills with performance appraisals and salary information in a Taiwanese high performance work organization. Unpublished Doctoral dissertation. University of Southern California.

Chetty, R., Friedman, J., Hilger, N., Saez, E., Whitmore Schanzenbach, D., \& Yagan, D. (2011). How does your kindergarten classroom affect your earnings? Evidence from Project STAR. Quarterly Journal of Economics, 126(4), 1593-660.

https://doi.org/10.1093/qje/qjr041

Davidson, K. (2016). Employers find 'soft skills' like critical thinking in short supply. The Wall Street Journal, 30. Recuperado de http://opportunityamericaonline.org/wpcontent/uploads/2016/08/EMPLOYERS-

FIND-\%E2\%80\%98SOFT-

SKILLS\%E2\%80\%99-LIKE-CRITICALTHINKING-IN-SHORT-SUPPLY.pdf

Delgado-Abella, L. E., León Rodríguez, V. L., Pinzón Ruiz, A. V., \& Marín Contreras, A. M. (2020) Diseño y propiedades psicométricas del Inventario para Evaluar la Efectividad de los Equipos de Trabajo (IET). Revista Iberoamericana de Diagnóstico y Evaluación - e Avaliação Psicológica, en prensa. https://doi.org/10.21865/RIDEP53.4.01

Deming, D. (2009). Early childhood intervention and life-cycle skill development: Evidence from head start. American Economic Journal: Applied Economics, 1(3), 111-34.

Deming, D. J. (2017). The value of soft skills in the labor market. NBER Reporter, (4), 7-11. https://doi.org/10.1257/app.1.3.111

Dieste, S. A., López, M. R., \& Martín, M. D. R. R. (2019). Percepciones de estudiantes universitarios sobre una evaluación formativa en el trabajo en equipo. Revista Iberoamericana de Evaluación Educativa, 12(1), 175-192.

Ferrando, P. J., \& Anguiano, C. (2010). El análisis factorial como técnica de investigación en psicología. Papeles del Psicólogo, 31(1), 1833.

Forero, C. G., Maydeu-Olivares, A., \& GallardoPujol, D. (2009). Factor analysis with ordinal indicators: A monte Carlo study comparing DWLS and ULS estimation. Structural Equation Modeling, 16, 625-641. https://doi.org/10.1080/10705510903203573 
Friedlaender, D., Burns, D., Lewis-Charp, H., Cook-Harvey, C. M., Zheng, X., \& DarlingHammond, L. (2014). Student-centered schools: Closing the opportunity gap. Stanford Center for Opportunity Policy in Education.

García, E. (2016). The need to address noncognitive skills in the education policy agenda. En M.S. Khine y S. Areepattamannil (Eds.), Non-cognitive skills and factors in educational attainment (pp. 31-64). Rotterdam: SensePublishers. https://doi.org/10.1163/9789463005913_004

Gates, S., Lippman, L., Shadowen, N., Burke, H., Diener, O., \& Malkin, M. (2016). Key soft skills for cross-sectoral youth outcomes. USAID's YouthPower: Implementation, YouthPower Action. Recuperado de https://www. fhi360. org/sites/default/files/media/documents/resour ce-soft-skills-report. pdf.

González-Morales, D., De Antonio, L. M. M., \& García, J. L. R. (2010). Teaching "Soft" skills in software engineering. 2011 IEEE Global Engineering Education Conference (EDUCON) (pp. 630-637). https://doi.org/10.1109/EDUCON.2011.5773204

Gutman, L., \& Schoon, I. (2013). The impact of non-cognitive skills on outcomes for young people: Literature review. Institute of Education, University of London. Recuperado de

https://pdfs.semanticscholar.org/f4a5/2db3001 fb6fb22eef5dc20267b5b807fd8ff.pdf

Hambleton R. K. (1996). Adaptación de tests para su uso en diferentes idiomas y culturas: Fuentes de error, posibles soluciones y directrices prácticas. En: Muñiz, J. (Ed.), Psicometría (pp. 207-238). Universitas.

Heckman, J., \& Kautz, T. (2013). Fostering and measuring skills: Interventions that improve character and cognition. National Bureau of Economic Research (Working Paper No. 19656). https://doi.org/10.3386/w19656

Hsieh, G. (2001). Types of feedback in a computer-based collaborative problemsolving group task. Unpublished doctoral dissertation. University of Southern California.
Hsieh, I. L., \& O’Neil, H. F. (2002). Types of feedback in a computer-based collaborative problem-solving group task. Computers in Human Behavior, 18, 699-715.

Hu, L. T., \& Bentler, P. M. (1999). Cutoff criteria for fit indexes in covariance structure analysis: Conventional criteria versus new alternatives. Structural Equation Modeling, 6(1), 1-55.

https://doi.org/10.1080/10705519909540118

Kautz, T., Heckman, J. J., Diris, R., Ter Weel, B., \& Borghans, L. (2014). Fostering and measuring skills: Improving cognitive and non-cognitive skills to promote lifetime success (No. w20749). National Bureau of Economic

Research. https://doi.org/10.3386/w20749

Kuehl, M. (2001). Revision of teamwork questionnaire for the United States Marine Corps aviation community. Unpublished doctoral dissertation. University of Southern California: Los Angeles.

Kyllonen, P. C. (2015). Designing test to measure personal attibutes and noncognitive skills. En S. Lane, M. R. Raymond, T. M. Haladyna (Eds.), Handbook of test development (pp.190-211). Routledge.

Ledesma, R. D., Ferrando, P. J., \& Tosi, J. D. (2019). Uso del Análisis Factorial Exploratorio en RIDEP. Recomendaciones para autores y revisores. Revista Iberoamericana de Diagnóstico y Evaluación - e Avaliação Psicológica, 52(3), 173-180. https://doi.org/10.21865/RIDEP52.3.13

Marshall, L., O’Neil, H. F., Chen, A., Kuehl, M., Hsieh, I., \& Abedi, J. (2005). Teamwork skills: Assessment and instruction. En J. Spector, C. Ohrazda, A. Schaak, \& D. Wiley (Eds.), Innovations in Instructional Technology (pp. 100-121). Lawrence Erlbaum Associates.

Matteson, M. L., Anderson, L., \& Boyden, C. (2016). "Soft skills": A phrase in search of meaning portal. Libraries and the Academy 16(1), 71-88.

https://doi.org/10.1353/pla.2016.0009

Muñiz, J., Elosúa, P., \& Hamblenton, R. K. (2013). Directrices para la traducción y adaptación de los tests: Segunda edición. Psicothema, 25, 151-157. 
Nagaoka, J., Farrington, C. A., Ehrlich, S. B., \& Heath, R. D. (2015). Foundations for young adult success: A developmental framework. concept paper for research and practice. University of Chicago Consortium on Chicago School Research.

O’Neil, H. F., Allred, K., \& Baker, E. L. (1997). Review of workforce readiness theoretical frameworks. In H.F. O'Neil (Ed.), Workforce Readiness: Competencies and assessment (pp. 3-5). Lawrence Erlbaum Associates.

O’Neil, H. F., Lee, C., Wang, S., \& Mulkey, J. (1999). Final report for analysis of teamwork skills questionnaire. Advanced Design Information.

O’Neil, H. F., Wang, S., Chung, G., \& Herl, H. (2000). Assessment of teamwork skills using computer-based teamwork simulations. En H.F. O'Neil \& D. Andrews (Eds.), Aircrew training and assessment (pp. 283-303). Lawrence Erlbaum Associates.

O’Neil, H. F., Wang, S., Lee, C., Mulkey, J., \& Baker, E. (2003). Assessment of teamwork skills via a teamwork questionnaire. En H.F. O’Neil \& R. Pérez (Eds.), Technology applications in education: A learning view. (pp. 283-301). Lawrence Erlbaum Associates.

Orbell, S., \& Verplanken, B. (2015). The strength of habit. Health psychology review, 9(3), 311317. https://doi.org/10.1080/17437199.2014.992031

Organización para la Cooperación y el Desarrollo Económicos (OCDE) (2015). Skills for Social Progress: The Power of Social and Emotional Skills. OCDE.

Penzenstadler, B., Mahaux, M., \& Heymans, P. (2013). University meets industry: calling in real stakeholders. En Software Engineering Education and Training (CSEE\&T 2013). IEEE 26th Conference on, (pp. 1-10). IEEE. https://doi.org/10.1109/CSEET.2013.6595231

Price, J. H., \& Murnan, J. (2004). Research limitations and the necessity of reporting them. American Journal of Health Education, 35, 66-67. https://doi.org/10.1080/19325037.2004.10603 611

Robles, M. M. (2012). Executive perceptions of the top 10 soft skills needed in today's workplace. Business Communication Quarterly, 75(4), 453-465. https://doi.org/10.1177/1080569912460400

Silva Carreño, W. H., \& Mazuera Moreno, J. A. (2019). ¿Enfoque de competencias o enfoque de capacidades en la escuela? Revista electrónica de investigación educativa, 21, e17, 1-10.

http://dx.doi.org/10.24320/redie.2019.21.e17. 1981

Steiger, J. H. (2007). Understanding the limitations of global fit assessment in structural equation modeling. Personality and Individual Differences, 42(5), 893-898. https://doi.org/10.1016/j.paid.2006.09.017

Verplanken B., \& Orbell, S. (2003). Reflections on past behaviour: A self-report index of habit strength. Journal of Applied Social Psychology, 33, 1313-1330. https://doi.org/10.1111/j.15591816.2003.tb01951.x

Verplanken B. (2006). Beyond frequency: Habit as mental construt. British Journal of Social Psychology, 45(3), 639-656. https://doi.org/10.1348/014466605X49122 